\title{
AC-DC Converter with Asymmetrical Higher Harmonics Compensation Function in Sustainable AC Grid
}

\author{
Grzegorz Wrona (Doctoral Student, Warsaw University of Technology), \\ Marek Jasinski (Assistant Professor, Warsaw University of Technology)
}

\begin{abstract}
In this paper the grid current higher harmonic compensation method for two level AC-DC converters in sustainable AC grid is described. The algorithm is based on Direct Power Control with Space Vector Modulator (DPC-SVM), which was extended by additional Higher Harmonics Compensation (HHC) module. The HHC block is composed of several resonant filters tuned to appropriate frequencies. Both, symmetrical and asymmetrical types of grid currents distortions are analyzed. Operation of the HHC module in stationary $\alpha \beta$ coordinates and rotating $d q$ coordinates is discussed. Selected waveforms illustrating operation of the developed algorithm are presented. It is shown that in $d q$ coordinates asymmetrical voltage distortions needs additional effort for its compensation in comparison to symmetrical higher harmonics. The subject is important because in practice asymmetrical higher harmonics usually can appear when one phase nonlinear load is connected to the grid. Even within such conditions the grid connected ACDC converter (GCC) should provide sinusoidal-like current. This assumption provides the reduction of negative impact of the GCC in power quality in Point of Common Coupling (PCC) in sustainable AC grid.
\end{abstract}

Keywords - Power quality, AC-DC power converters, pulse width modulation inverters, bidirectional power flow, power harmonic filters.

\section{INTRODUCTION}

In recent years there have been several issues related to sustainable future Electrical Grids that should be taken into consideration. From one side it is evident that DC grid will be developed and redesigned [1]. However, it has to be stressed that AC grid will also be improved i.e. its reliability, power quality and controllability. Taking this into account, power electronics AC-DC converter control should provide a lot of functions in sustainable energy conversion systems. Because this subject is wide, it is not possible to present all power quality problems (voltage dips, swells, flickers, notches etc.) and solutions for their elimination in a short paper. Here only the higher harmonic compensation will be presented. Because this subject is quite well described in the literature [2], [3], [4] in this paper the analysis of higher harmonics distortion will be extend to the case of the asymmetrical deformation caused by a single phase load connected to PCC. From the grid point of view it is most important to fulfill power quality requirements [5]. These requirements can be improved by dedicated control of an AC-DC converter. Because the number of Renewable Energy Sources (RES) are continuously growing it is important to improve the control methods related with grid connected AC-DC converters (GCC). This control can assure more stable operation of GCC converters and moreover, it can improve the current quality (indirectly grid voltage quality) in Point of Common Coupling (PCC) distorted by single phase nonlinear loads.

Higher harmonics increase losses in transformers and transmission lines and can shorten their lifetime.

In this paper the current asymmetrical higher harmonics compensation will be implemented in Direct Power Control with Space Vector Modulation (DPC-SVM) [3], [6] and [7].

Among higher harmonics compensation methods there are the three most common which are based on:

- repetitive control [8],

- Multiple Synchronous Reference Frames (MSRF) [9],

- Generalized Integrators (GI) [10].

All methods are based on extraction of harmonics from fundamental component. Extracted signals are summed and added to the converter reference voltage forming additional control loops.

In this paper the basics of operation of the GCC, proposed control algorithm and Generalized Integrator (GI) is presented (Chapter II). Moreover, the basic principles related with higher harmonics in three phase system are shown. Then in Chapter III harmonics compensation for the three phase, balanced system is described shortly. Additionally in Chapter IV the principles of operation in balanced and unbalanced three phase system are explained. Finally in Section V the selected simulations and experimental results under unbalanced higher harmonics conditions are shown. Summary and conclusions are provided at the end.

\section{OPERATION OF THE GRID CONNECTED CONVERTER UNDER HIGHER HARMONICS DISTORTION}

The phenomenon of higher harmonics is related to both currents and voltages. However due to the grid and AC filter impedance they are closely related. Fig.1 shows the sample section of the grid system with a set of various devices connected to it. One of the devices is high power, highly nonlinear load. This device generates strongly distorted current. Due to the impedance of the transmission line, this current affects the voltage in the Point of Common Coupling (PCC) causing its distortion as it is presented in Fig.1. 


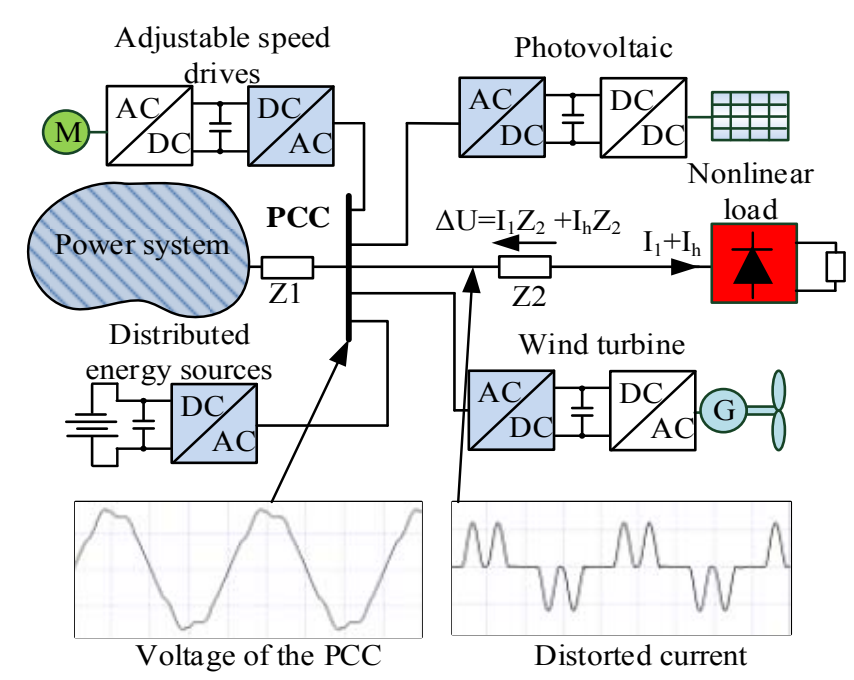

Fig.1. The sample section of the grid system with a set of various devices and a high power nonlinear load.

Since the voltage in PCC is distorted, other devices connected to the PCC are influenced by the distortion. If GCCs are not properly adapted to work in such conditions they will operate improperly generating non sinusoidal currents thereby aggravating distortion or they may be damaged. Therefore, all devices connected to the grid should be able to withstand higher harmonic distortion. However, the most popular control methods for AC-DC Converter (GCC) such as Voltage Oriented Control VOC or Direct Power Control DPC-SVM [6] are not suitable to work in such condition. In this paper additional higher harmonic compensation HHC module for GCC control will be introduced. Theoretical, simulation and experimental analysis based on DPC-SVM control method will be carried out.

The model of GCC can be described in different coordinate system. The basic scheme of the GCC with AC input choke and output DC side capacitor is shown in Fig.2(a), while Fig.2(b) shows its single-phase representation. Where, $\mathbf{U}_{\mathrm{L}}$ is the line voltage complex space vector, $\mathbf{I}_{\mathrm{L}}$ is the line current space vector, $\mathbf{U}_{\mathrm{P}}$ is the GCC input voltage space vector, and $\mathbf{U i}$ is the space vector of voltage drop on the $\mathrm{AC}$ grid side choke $\mathrm{L}$ and its resistance $\mathrm{R}$ (Fig.3).

The Up voltage is controllable and depends on the switching signals pattern and DC-link voltage level. Thanks to control magnitude and phase of the Up voltage, the line current can be controlled by changing the voltage drop on the input choke - Ui. Therefore, inductances between grid and AC side of the GCC are indispensable. They create a current source and provide boost feature of the GCC. Through controlling the converter AC side voltage in its phase and amplitude $\mathbf{U p}$, the phase and amplitude of the line current vector $\mathbf{I}_{\mathrm{L}}$ is controlled indirectly. For the sinusoidal voltages, the line current can be described using the complex space vector notation as:

$$
\mathbf{I}_{L}=\frac{U_{L} e^{j \gamma}-U_{p} e^{j(\gamma+\varphi)}}{R+j \omega L},
$$

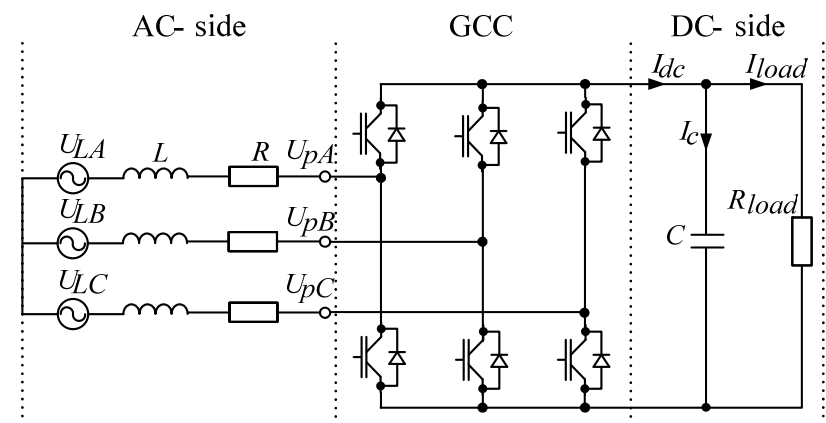

(a)

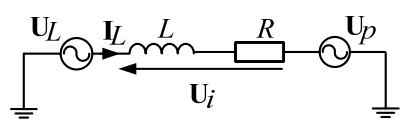

(b)

Fig.2. AC-DC Converter topology: (a) three phase system, (b) single phase equivalent.

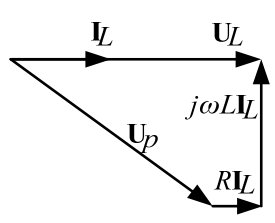

a)

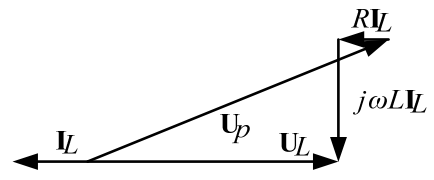

b)
Fig.3. Phasor diagrams. The AC-DC Converter operates with unity power factor operation: (a) rectifying mode, (b) inverting mode.

However, the distorted grid voltage with $\mathrm{k}$ harmonics can be expressed as:

$$
\mathbf{U}_{L}=U_{1} e^{j \gamma}+\sum_{k=2}^{\infty} U_{k} e^{ \pm j k \gamma},
$$

where: $\gamma=\omega \mathrm{t} ; \mathrm{k}=2,3 \ldots$

\section{A. Principles of Higher Harmonics Distortion in the Three Phase System}

There is a simple relationship between the order of the harmonics occurrence in three-phase balanced (symmetrical distortion in each phase) circuits and the corresponding sequence of phases:

- positive sequence harmonics can be calculated from the following equation: $3 \mathrm{k}+1(1,4,7,10 \ldots)$,

- negative sequence harmonics can be calculated from the following equation: $3 \mathrm{k}+2(2,5,8,11 \ldots)$,

- zero sequence harmonics are expressed as: $3 \mathrm{k}+3(3,6,9,12 \ldots)$.

Periodic signals satisfying half-wave symmetry property can contain only odd harmonics. In addition, in the threephase, three-wire circuits, zero sequence components are absent. According to this assumptions, the higher harmonics to be compensated are expressed as: $6 \mathrm{k}+/-1$, where: $\mathrm{k}=1,2,3 \ldots$ and the most prominent harmonics in a typical higher

where: $\gamma=\omega t$. 
harmonics spectrum of the grid current are: 5th, 7th, 11th, 13 th.

\section{B. Proposed Control Algorithm with Higher Harmonics Compensation for the Three Phase System}

Block scheme of the control method is presented in Fig 4. The operation of the algorithm relay on the estimated instantaneous active and reactive power calculated as in [11] and [12]:

$$
\begin{gathered}
P=\left(U_{L \alpha} I_{L \alpha}+U_{L \beta} I_{L \beta}\right) \text { and } \\
Q=\left(U_{L \alpha} I_{L \beta}-U_{L \beta} I_{L \alpha}\right) .
\end{gathered}
$$

The estimated active and reactive power is compared with commanded values and the instantaneous errors at PI controller outputs determine converter voltage. After transformation to the stationary coordinates $\alpha \beta$, these signals are delivered to the Space Vector Modulator block (SVM). To meet unity power factor condition, reactive power reference value is set to zero, while the active power is controlled by the DC-link voltage PI-controller. There is a possibility to control the value of reactive power, which is important in the case of voltage dips. A low pass filter was used to eliminate the influence of the DC voltage ripples caused by the harmonics. The actual angle of the grid voltage is given by phase locked loop PLL [13] module.

Basic DPC-SVM control method operates properly ensuring sinusoidal current only with sinusoidal grid voltage. When the higher harmonics appear, generated current is also strongly distorted leading to a system instability or even failure. Therefore additional Higher Harmonics Compensation (HHC) module is required to feed sinusoidal-like current.

Adaptation of the HHC module for the DPC-SVM relies on the extraction of harmonics from the fundamental components by the use of Generalized Integrator (GI). Compensation can be realized in stationary $\alpha \beta$ coordinates $\left(U_{\alpha h}, U_{\beta h}\right)$ as well as in rotating coordinates $\left(U_{d h}, U_{q h}\right)$ which will be described later in this work.

At the input of the HHC module there are the measured grid currents. The outputs, compensation signals from the compensator are added to the main control loops.

\section{Description of the Generalized Integrator}

Currently one of the most efficient compensation methods is based on the Generalized Integrator (GI) which performs function of a pass band filter with very narrow bandwidth. The transfer function of an ideal GI is defined as:

$$
U_{h}(s)=\sum_{h=5,7,11,13} K_{i} \frac{s}{s^{2}+(h \omega)^{2}},
$$

where: $\mathrm{K}_{\mathrm{i}}$-gain, $\omega$ - frequency of the fundamental component, h- harmonic row.

Bode diagram of the GI tuned to $250 \mathrm{~Hz}$ frequency is presented in Fig.5. It can be seen that an ideal integrator has very high gain (infinite) and narrow pass band around the resonant frequency. This allows extracting selected harmonics from the measured signals in a very selective way.

GI is a resonant structure that performs a function of the integrator for sinusoidal signals and it is described in [10] and
[14]. This structure is also a fundamental block of the control system known as Proportional Resonant (PR) controllers [15].

Modern control systems are usually realized as digital systems and implemented on digital platforms, i.e.: DSP or FPGA [7]. For this purpose, it is required to find the discrete equivalent of a continuous system. Prewarped bilinear transformation from continuous-time representations to discrete-time representation (also known as prewarped Tustin's method) was used. This technique is the most commonly used digitization technique and relies on substitutions (6) into (5).

$$
s=K \cdot \frac{z-1}{z+1}
$$

where $K=\omega / \operatorname{tg}(\omega T s / 2)$.

The result is a discrete transfer function of the following form:

$$
T(z)=\frac{a_{0} z^{2}+a_{1} z+a_{2}}{b_{0} z^{2}+b_{1} z+b_{2}} .
$$

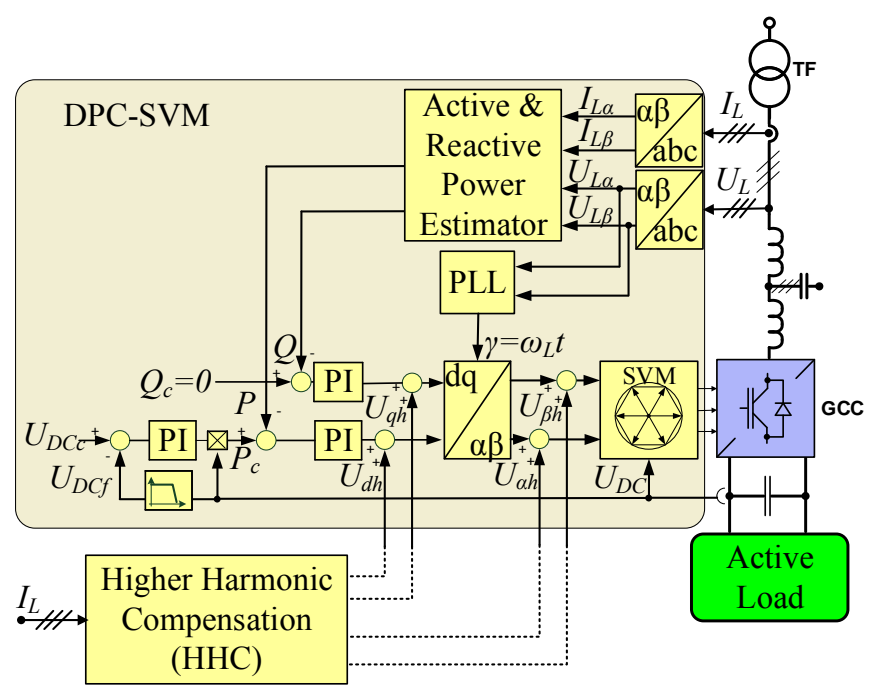

Fig.4. Block scheme of the control method.



Fig.5. Bode diagram of the ideal Generalized Integrator (GI) tuned to $5^{\text {th }}$ harmonic $(250 \mathrm{~Hz})$ for different gain $(\mathrm{Ki})$ factors. 
In (7) the respective coefficients are equal to:

$$
\begin{aligned}
& a_{0}=0 \\
& a_{1}=2 \omega_{c} K \\
& a_{2}=-2 \omega_{c} K \\
& b_{0}=\omega^{2}+2 \omega_{c} K+K^{2} \\
& b_{1}=\omega^{2}-2 K^{2} \\
& b_{2}=\omega^{2}-2 \omega_{c} K+K^{2} .
\end{aligned}
$$

After substituting $\omega_{c}=1, T s=0.0002, \omega=300$ and $\omega=600$ into equations (7) and (8) results were obtained:

$$
\begin{gathered}
T_{300}(z)=K_{i} \frac{0.0001953 z-0.0001953}{z^{2}-1.859 \cdot z+0.9996} \text { and } \\
T_{300}(z)=K_{i} \frac{0.0001815 z-0.0001815}{z^{2}-1.458 \cdot z+0.9996} .
\end{gathered}
$$

Online calculation of the filter coefficients increases the complexity of the algorithm, which can be a problem in implementation on a digital system; however, it allows maintaining the filter properties and performance at a higher range of frequencies. This approach is based on the substitution of the actual value of the frequency obtained from PLL module into equations (6) and (8).

\section{Higher HARMONiCS COMPENSATION IN THE THREE PHASE BALANCED SYSTEM}

\section{A. Compensation in the Stationary $\alpha \beta$ Coordinates}

The idea of positive and negative sequence harmonics described in the Chapter II is presented in Fig.6. The vector diagram shows the $1^{\text {st }}, 5^{\text {th }}, 7^{\text {th }}, 11^{\text {th }}$ and $13^{\text {th }}$ harmonics in $\alpha \beta$ coordinate and their rotating directions. For the analysis of the harmonic spectrum, the Fast Fourier Transform is used. However FFT does not represent harmonic sequence. Therefore modified harmonic spectrum with spread positive and negative sequence is presented in Fig.7. This will be useful in the analysis.

In the three-phase balanced system, the grid current with higher harmonics, in stationary $\alpha \beta$ reference frame, can be expressed as:

$$
\mathbf{I}_{L}=I_{1} e^{j \gamma}+I_{5} e^{-j 5 \gamma}+I_{7} e^{j 7 \gamma}+\ldots+I_{(6 k \pm 1)} e^{j_{(6 k \pm 1)} \gamma},
$$

where: $\mathbf{I}_{L^{-}}$grid voltage, $I_{\mathrm{n}}$-is module of the $\mathrm{n}$-th harmonic,

$$
\gamma=\omega t ; k=2,3 \ldots
$$

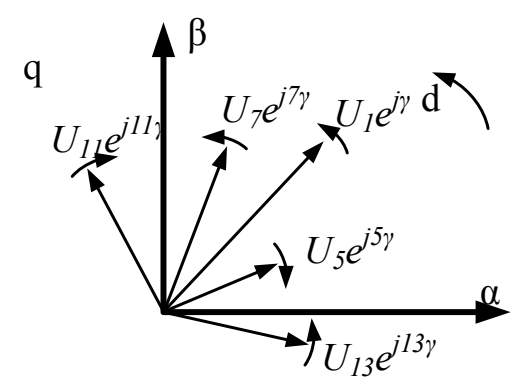

Fig.6. Principle illustration of positive and negative sequence components based on 5th, 7th, 11th and 13th harmonics.

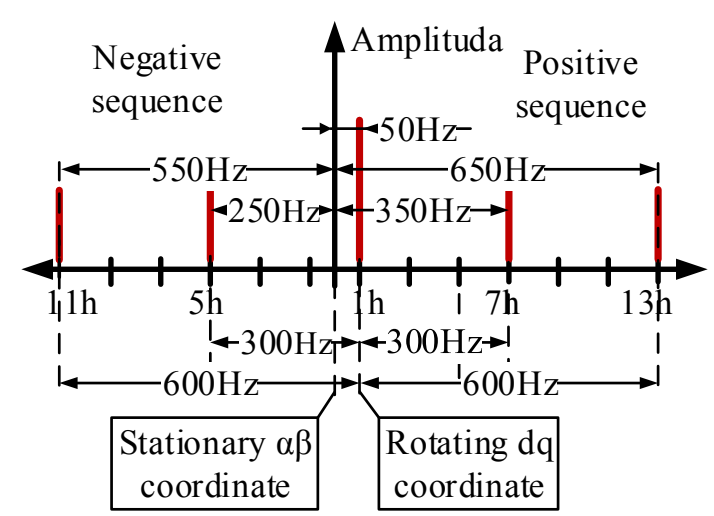

Fig.7. Harmonics spectrum with spread positive and negative sequence components based on 5th, 7th, 11th and 13th harmonics.

The block diagram of the HHC in $\alpha \beta$ coordinates is presented in Fig.8. Each harmonic is compensated separately by one regulator tuned to appropriate frequency specified by the formula $6 \mathrm{k}+/-1$. Therefore in the control structure there will be as much regulators as higher harmonics being compensated.

\section{B. Higher Harmonics Compensation in the Rotating $d q$ Coordinates}

Due to the transformation from stationary $\alpha \beta$ to rotating $d q$ reference frame, the frequency of the higher harmonics is going to be different. The distorted grid current in this case can be expressed as:

$$
\begin{array}{r}
\mathbf{I}_{L} e^{j \gamma}=I_{1}+I_{5} e^{-j 6 \gamma}+I_{7} e^{j 6 \gamma}+I_{11} e^{-j 12 \gamma}+ \\
I_{13} e^{j 12 \gamma}+\ldots,
\end{array}
$$

where: $\gamma=\omega t ; k=1,2, \ldots$.

According to the equation (12), $5^{\text {th }}$ and $7^{\text {th }}$ harmonics are visible with the same $300 \mathrm{~Hz}$ frequency but rotating in opposite directions. Therefore, these two harmonics can be compensated by single resonant filter tuned to appropriate frequency. This constitutes one of the main advantages of this compensation method. Similar relationship is for $11^{\text {th }}$ and $13^{\text {th }}$ harmonic.

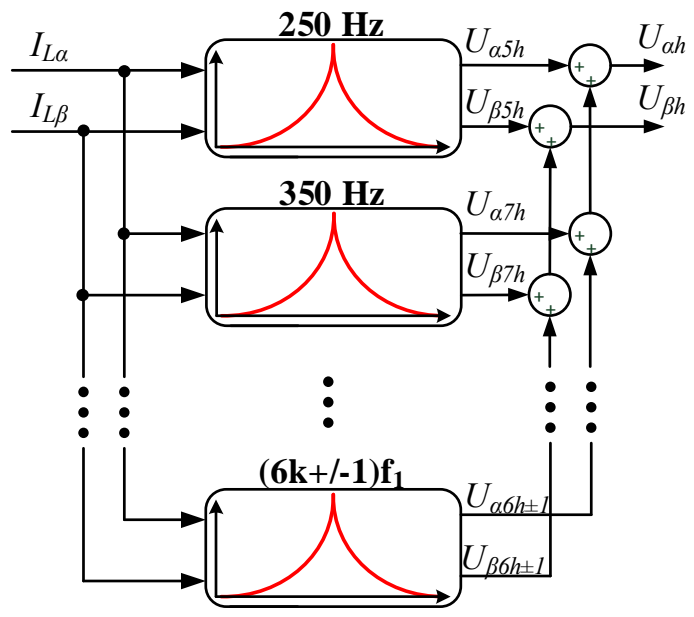

Fig.8. Block scheme of the HHC in $\alpha \beta$ coordinates. 


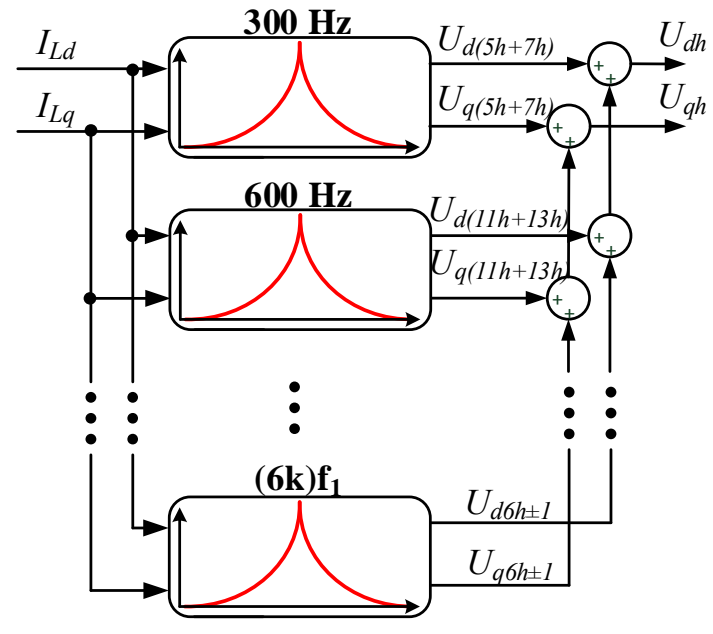

Fig.9. Block scheme of the HHC in dq coordinates.

In this case the frequency of each harmonic can be calculated as $+/-6 \mathrm{k}$. Therefore scheme of the harmonic compensation module has the form presented in Fig.9.

\section{Higher Harmonic Compensation in the Three Phase UNBALANCED SYSTEM}

The HCC algorithm described in previous chapter works well with symmetrical distortion in each phase. However, when the grid voltage is distorted asymmetrically e.g. higher harmonics in only one phase, this algorithm cannot operate properly. This phenomenon is very common and can be caused by high power single phase nonlinear loads connected to the grid or asymmetrical grid impedance. The theory of symmetrical components developed by Fortescue in [16] and [17] will be used for the analysis of the asymmetrical harmonics in the three phase unbalanced system. The key idea of the symmetrical component analysis is to decompose the system into three sequence networks. Fortescue proved that any set of unbalanced three-phase quantities could be expressed as the sum of three symmetrical sets of balanced phasors. Principles of the positive and negative networks were presented in the chapter III.

\section{$A$. Compensation in the stationary $\alpha \beta$ coordinates}

In the stationary $\alpha \beta$ reference frame by applying this methodology to each of the harmonics found in the grid current we can get following formula:

$$
\begin{aligned}
\mathbf{I}_{L}=I_{1} e^{j \gamma} & +I_{5 p} e^{-j 5 \gamma}+I_{5 n} e^{-j 5 \gamma}+I_{7 p} e^{j 7 \gamma}+ \\
& +I_{7 n} e^{j-7 \gamma}+I_{(6 k \pm 1) p} e^{j_{(6 k \pm 1)} \gamma}+I_{(6 k \pm 1) n} e^{-j_{(6 k \pm 1)} \gamma},
\end{aligned}
$$

where: $\gamma=\omega \mathrm{t} ; \mathrm{k}=2,3 \ldots$

The effect of this analysis is presented in Fig.10. The additional stripes in the harmonic spectrum are highlighted with a circle. Note that, the number of resonant filters will be the same as in balanced system since the harmonics with negative and positive angular speed has the same frequencies in $\alpha \beta$ coordinates.

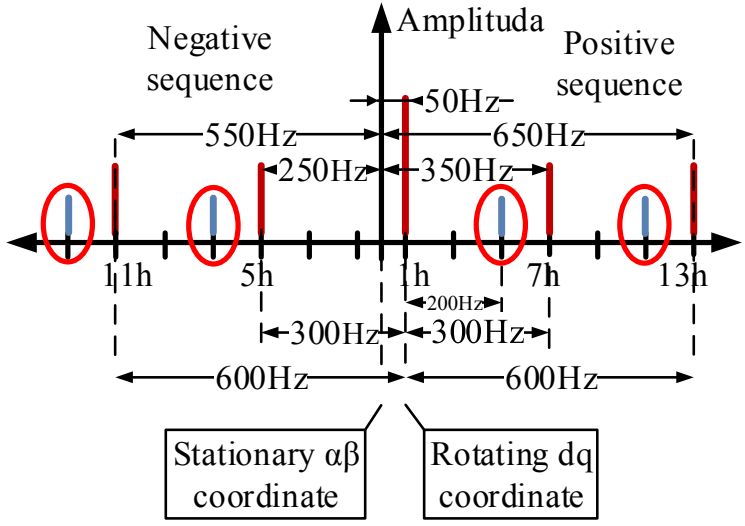

Fig.10. Harmonics spectrum with spread positive and negative sequence components based on 5th, 7th, 11th and 13th harmonics with additional negatives components.

TABLE I

MAIN PARAMETERS OF THE Simulation MODEL

Converter parameters

\begin{tabular}{l|l}
\hline \multicolumn{2}{c}{ Converter parameters } \\
\hline Switching frequency, Nominal Power PN & $5 \mathrm{kHz}, 55 \mathrm{kVA}$ \\
\hline DC link capacitor & $2.73 \mathrm{mF}$ \\
\hline Dead time & $2 \mu \mathrm{s}$ \\
\hline \multicolumn{2}{c}{ LCL Filter } \\
\hline Grid side inductance & $0.21 \mathrm{mH}$ \\
\hline Capacitance & $44 \mathrm{uF}$ \\
\hline Converter side inductance Measurement conditions \\
\hline \multicolumn{2}{c}{$0.97 \mathrm{mH}$} \\
\hline Phase Line voltage, frequency & $3 \times 230 \mathrm{~V}(\mathrm{rms}), 50 \mathrm{~Hz}$ \\
\hline DC link voltage & $700 \mathrm{~V}$ \\
\hline DC load & $15 \Omega$ \\
\hline
\end{tabular}

\section{B. Compensation in the rotating $d q$ coordinates}

Slightly different situation is for the asymmetrical harmonics compensation in $\mathrm{dq}$ reference frame. In this case the positive and negative components of each harmonic are defined by different frequencies.

For example, $5^{\text {th }}$ harmonic in the unbalanced system can be split into positive component $I_{5 p} e^{j 5 \gamma}$ and negative component $I_{5 n} e^{-j 5 \gamma}$. However, after transformation to $d q$ coordinates, in frequency domain, positive component is shifted down by the $\gamma$ factor, while negative component is shifted up by the same factor. Therefore each component will be represented by $200 \mathrm{~Hz}$ and $300 \mathrm{~Hz}$ frequencies in rotating reference frame.

\section{V.SELECTED SIMULATION AND EXPERIMENTAL RESULTS.}

The developed algorithm presented in Fig.4 was implemented and tested in the advanced simulation package Saber Synopsys. The parameters of the simulation model are shown in Table I. The model consists of two parts: power circuit (grid, step down transformer, LCL filter, AC-DC converter and active load) and signals circuits (control with measurements blocks). The control algorithm was written in MUST language. It gives a possibility for relatively fast laboratory implementation in $\mathrm{C}$ because the syntax of MUST and $\mathrm{C}$ language is quite similar. Selected simulation results are presented below. 

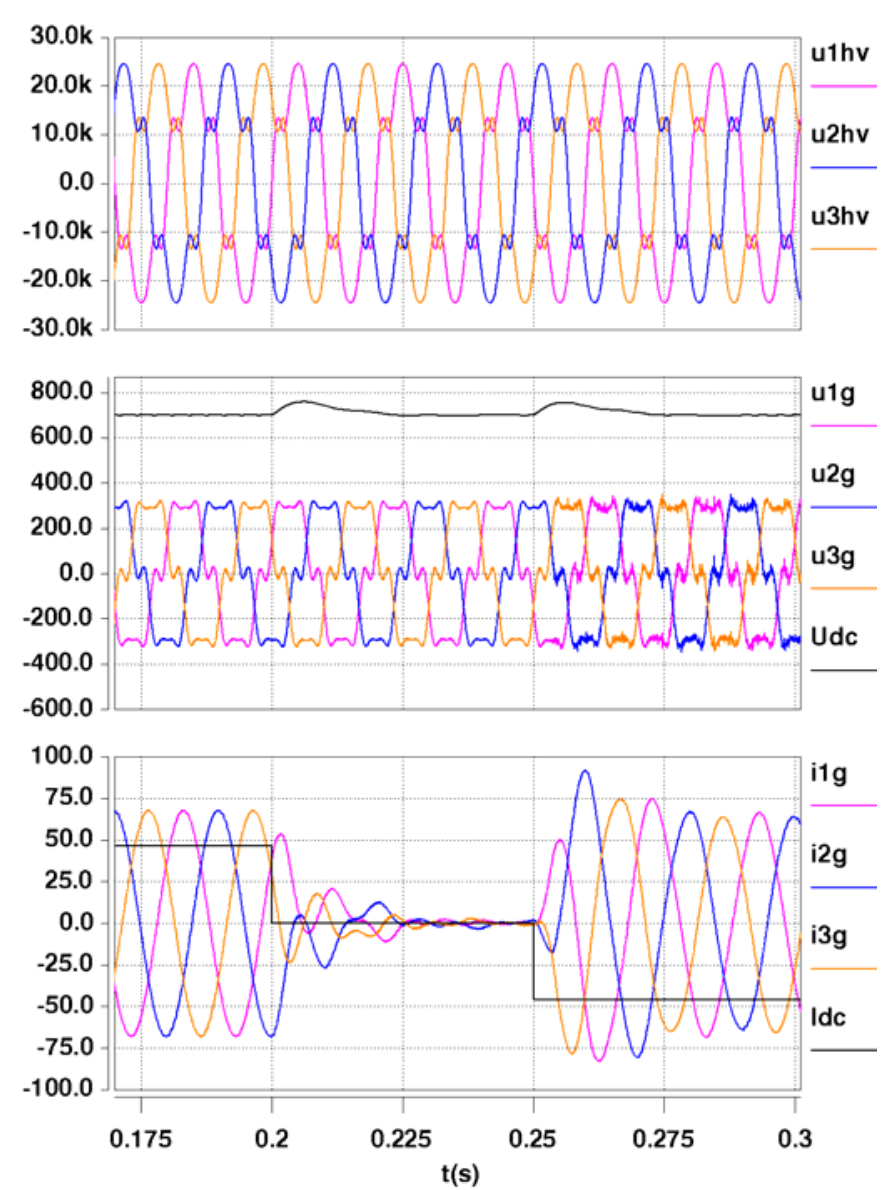

Fig.11. Transient states: three modes of operation with HHC module switched on under symmetrical grid voltage distortion - operation in dq coordinates (THDU $=20 \%$ of the 5 th harmonic). From the top: transformer voltages u1hv, u2hv, u3hv [V]; grid voltages u1g, u2g, u3g [V] and DC voltage Udc [V]; grid currents i1g, i2g, i3g [A] with DC current Idc[A].

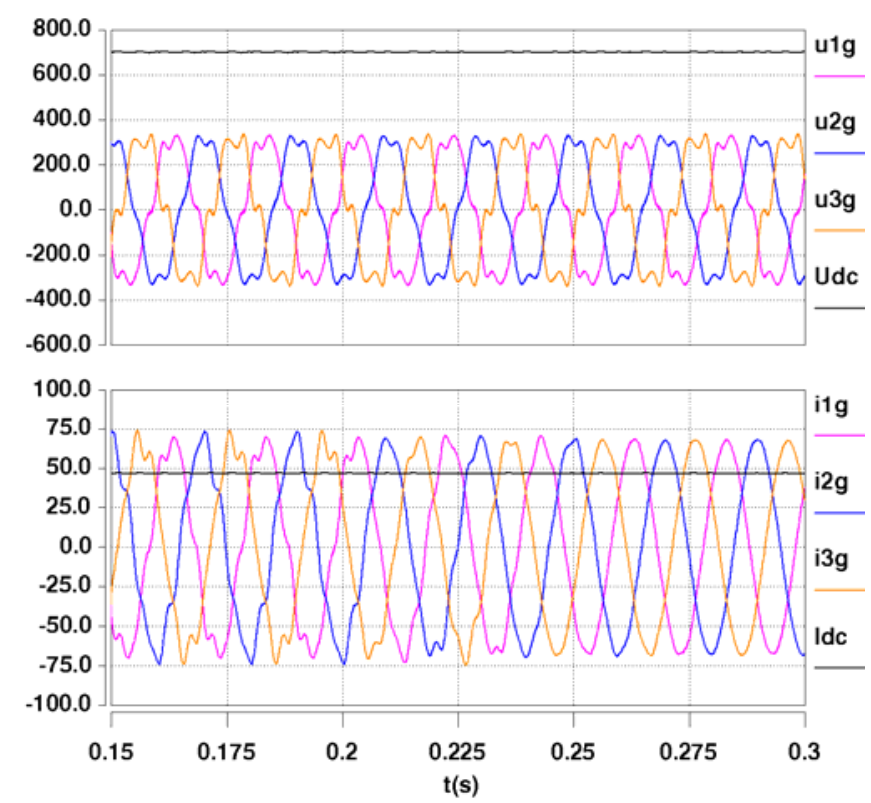

Fig.12. Transient states: three phases of operation: for $\mathrm{t}<0.2 \mathrm{~s} \mathrm{HHC}$ module is switched off, HHC module adopted to symmetrical distortion switched on $(\mathrm{t}=0.2 \mathrm{~s})$, HHC module adopted to asymmetrical harmonics with switched operation in dq coordination. From the top: grid voltages u1g, u2g, u3g [V] and DC voltage Udc [V]; grid currents i1g, i2g, i3g [A] with DC current Idc [A].

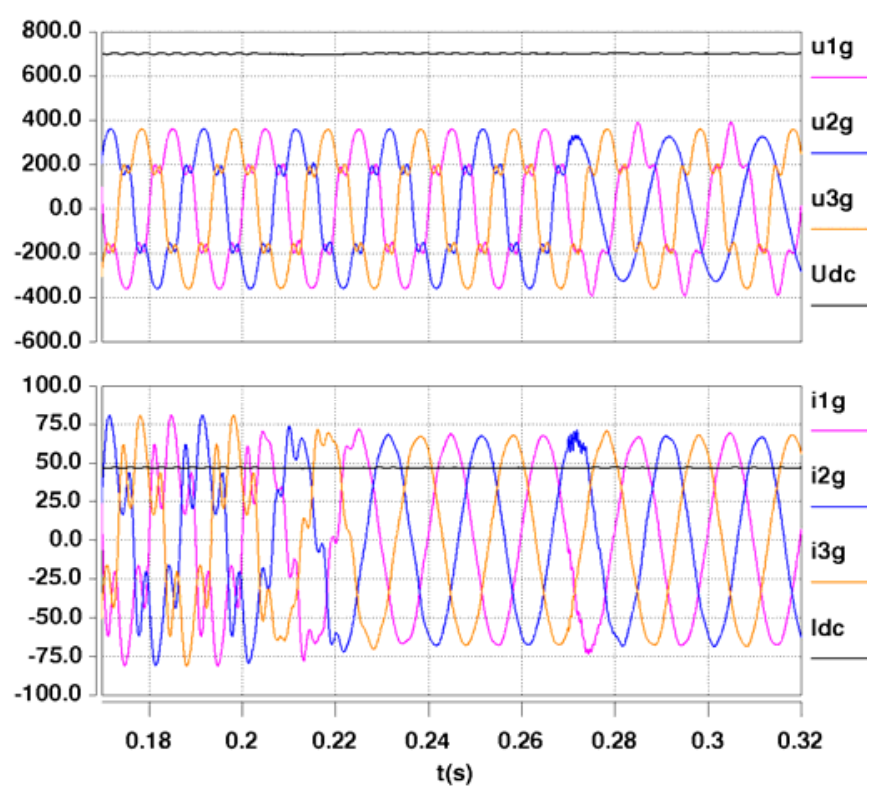

Fig.13. Transient states: three phases of operation: for $\mathrm{t}<0.2 \mathrm{~s}$ HHC module switched off, for $\mathrm{t}<0.28 \mathrm{~s}$ HHC module under symmetrical distortion switched on, HHC module under asymmetrical distortion - operation in $\alpha \beta$ coordination. From the top: grid voltages u1g, u2g, u3g [V] and DC voltage $\mathrm{Udc}$ [V]; grid currents i1g, i2g, i3g [A] with DC current Idc[A].

In the Fig.11 and Fig.12 operation of the HHC module within symmetrical and asymmetrical higher harmonics distortion is presented. Compensation is realized in rotating $\mathrm{dq}$ coordinates. Fig.11 shows three modes of operation: rectifying mode $(\mathrm{t}<0.2 \mathrm{~s})$ with the load of $15 \Omega$, idle mode $(0.2 \mathrm{~s}<\mathrm{t}<$ $0.25 \mathrm{~s})$ and inverting mode $(\mathrm{t}>0.25 \mathrm{~s})$ with DC current source set to $46 \mathrm{~A}$. In rectifying and inverting cases the $\mathrm{THD}_{\mathrm{I}}$ is lower than $1 \%$. The grid voltage is symmetrically distorted by $5^{\text {th }}$ $(20 \%)$ and $7^{\text {th }}(10 \%)$ harmonics. Due to the step down transformer, the distortion on the low voltage side and high voltage side is slightly different. Operation of the compensation module with asymmetrical distortion is presented in Fig.12.

Before the time $\mathrm{t}=0.2 \mathrm{~s}$ only compensation for symmetrical distortion is in on state. It can be seen that the phase currents still contain significant amount of higher harmonics. After the time $\mathrm{t}=0.2 \mathrm{~s}$ additional (resonant filters tuned to $200 \mathrm{~Hz}$ and $400 \mathrm{~Hz}$ ) compensation was switched on. It can be seen that after three periods of the grid voltage the shape of the current is nearly sinusoidal.

Compensation in stationary $\alpha \beta$ reference is presented in Fig.13. At the time before $\mathrm{t}=0.2 \mathrm{~s}$ the compensation module is switched off. The current waveforms (i1g, i2g, i3g) are strongly distorted. Next the algorithm is enable in $\mathrm{t}=0.2 \mathrm{~s}$. At the time $\mathrm{t}=0.27 \mathrm{~s}$ asymmetrical higher harmonics $\left(20 \%\right.$ of $5^{\text {th }}$ in two phases and $10 \%$ of $7^{\text {th }}$ in one phase) are applied in grid voltages.

Compensated current for both symmetrical and asymmetrical distortion has sinusoidal shape with low harmonic content. 
To verify operation, the control algorithm has been tested on a $55 \mathrm{kVA}$ laboratory test bench. Parameters of the laboratory setup are presented in Table II, but the results - in Fig.14 and Fig.15. Note that, due to the presence of the GC transformer, the distortion may be different from given value. The effect of these variations can be observed in the Fig 15(c). The transformer voltage on the primary side contains $15 \%$ of $5^{\text {th }}$ harmonic in one phase, while on the secondary side, the transformer voltages are distorted in two phases

Fig.14 and Fig. 15 show the waveforms while GCC operates in rectifying mode with switched off and switched on harmonic compensation module respectively. The $\mathrm{HHC}$ module was implemented in dq coordinates. In the Fig.14a current waveforms generated by the converter under asymmetrical distortion of the grid voltage $\left(15 \%\right.$ of $5^{\text {th }}$ harmonic in one phase) is presented. It can be seen that the currents are strongly distorted $\left(\mathrm{THD}_{\mathrm{I}}=50.1 \%\right)$. Fig. $14(\mathrm{~b})$ shows their harmonic spectrum.

After the compensation, adapted to asymmetrical distortion, was enabled, the $\mathrm{THD}_{\mathrm{I}}$ fell to $3.2 \%$ what is presented in Fig 15(b).

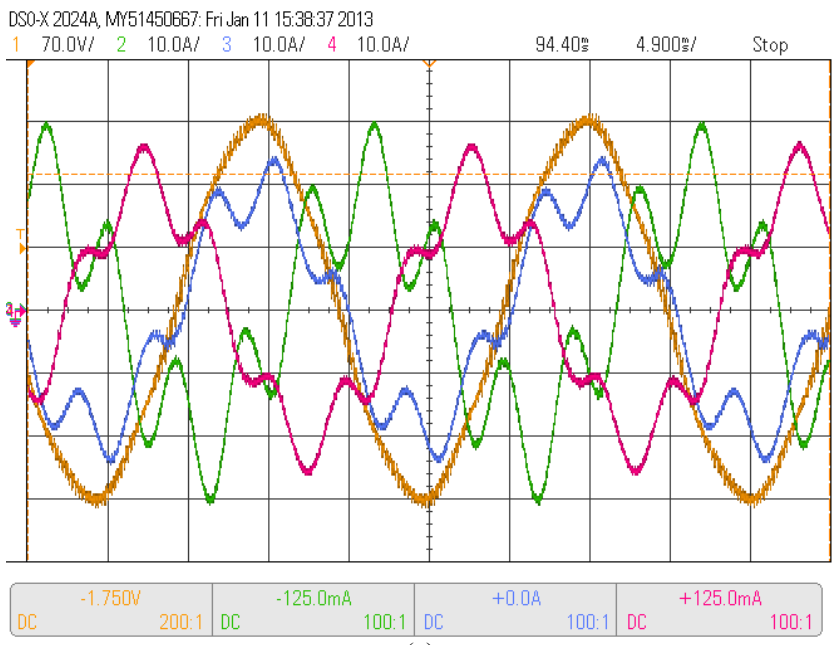

(a)

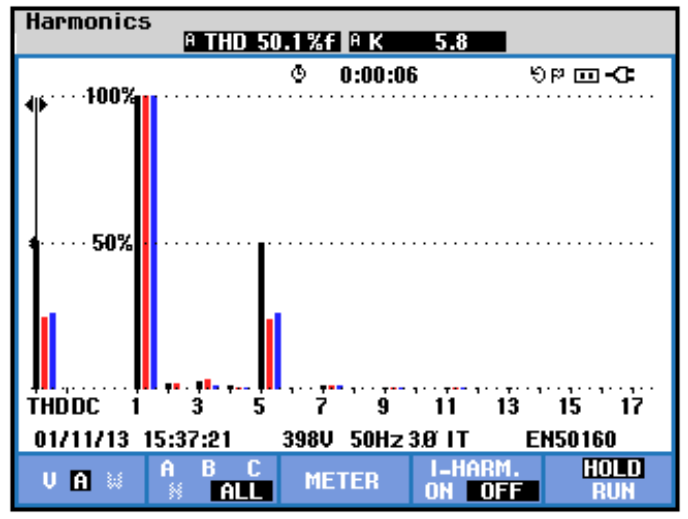

(b)

Fig.14. Steady states - operation of the DPC-SVM control algorithm during grid voltage distortion with switched off HHC module: (a) waveforms of the three phase currents and phase voltage, (b) three-phase currents harmonic spectrum. In the oscillogram: grid voltage [70 V/div], but three phase currents [10 A/div]
TABLE II

MAIN PARAMETERS OF THE LABORATORY SETUP

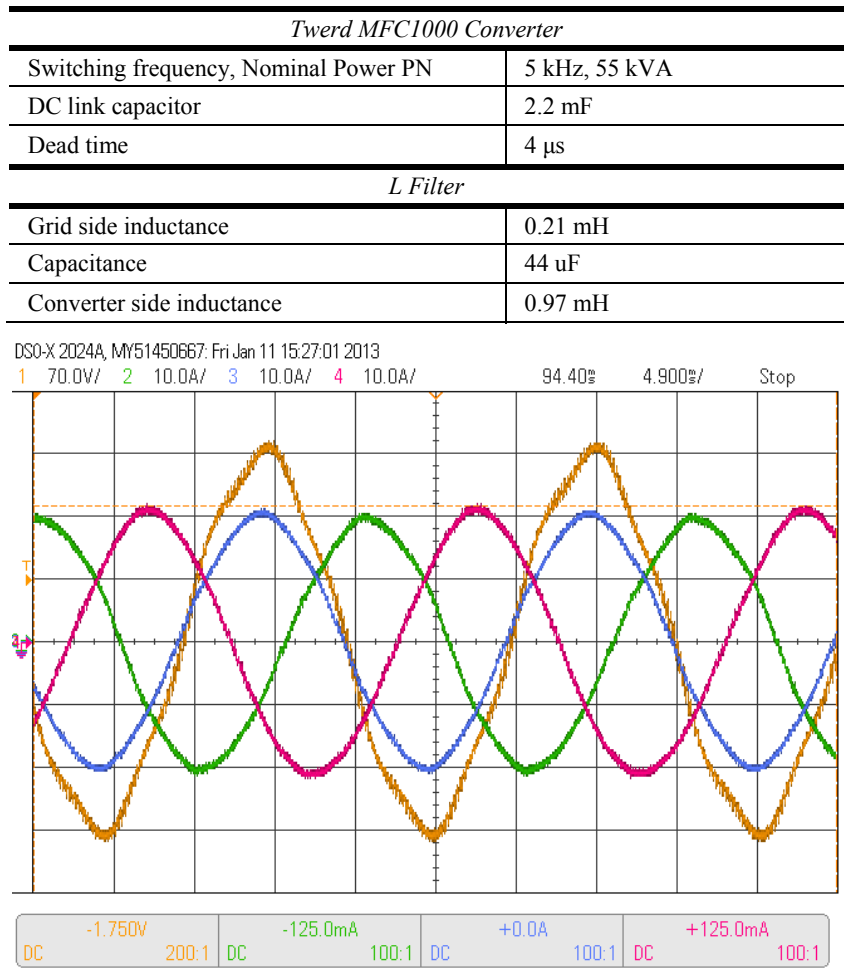

(a)

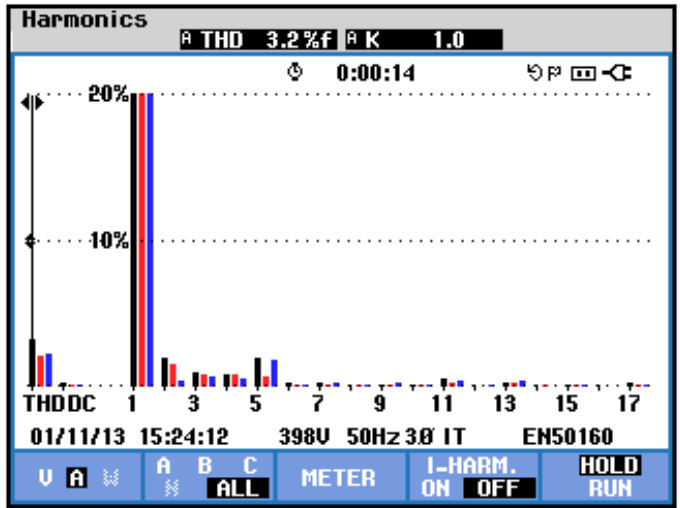

(b)

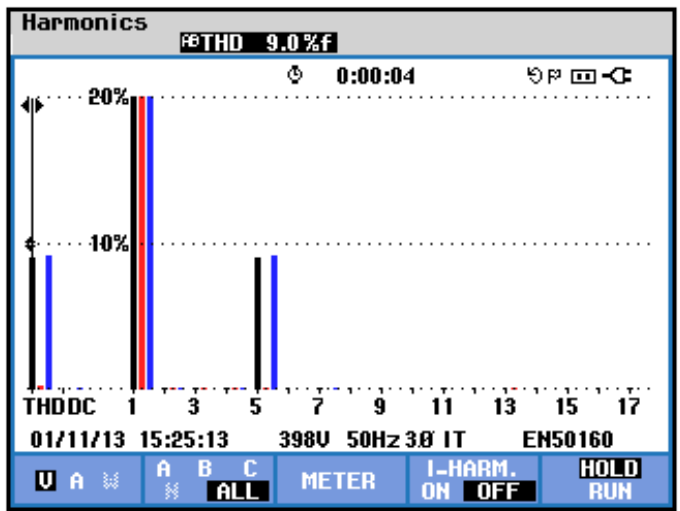

(c)

Fig.15. Steady states - operation of the DPC-SVM control algorithm during grid voltage distortion with switched on HHC module: (a) waveforms of the three phase currents and phase voltage, (b) three phase currents harmonic spectrum, (c) three phase voltages harmonic spectrum. In the oscillogram: grid voltage $[70 \mathrm{~V} / \mathrm{div}]$, but three-phase currents $[10 \mathrm{~A} / \mathrm{div}]$. 
DSO-X 2024A, Mr51450667: Wed 0ct 31 16:42:07 2012

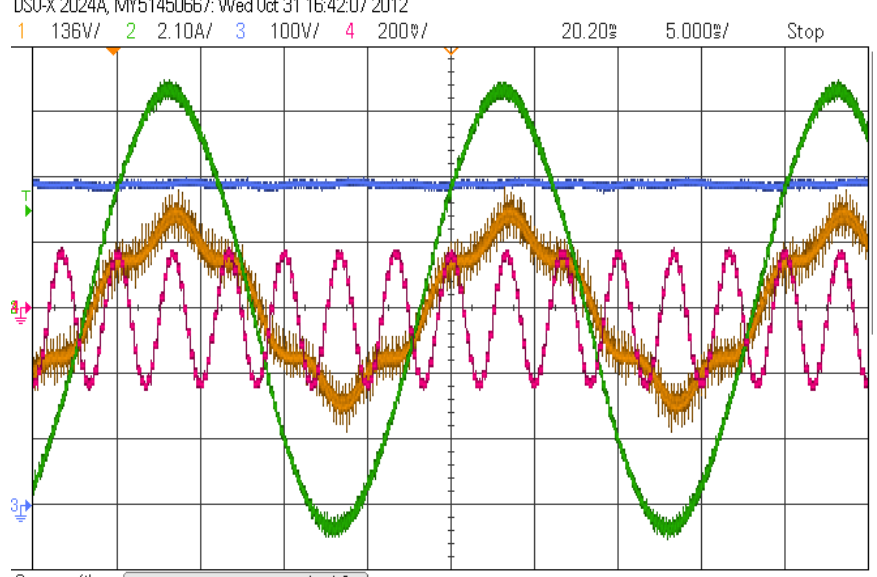

Fig.16. Operation of the DPC-SVM control algorithm under three phase symmetrical distortion with HHC module implemented in $\alpha \beta$ coordinates. From the top (green- grid current, blue - DC link voltage, yellow - grid voltage, purple - compensation signal).

Fig.16 shows operation of the compensation implemented in $\alpha \beta$ coordinates. Grid voltage is symmetrically distorted by $15 \%$ of $5^{\text {th }}$ harmonic. It can be seen that the grid current higher harmonics were reduced significantly.

\section{CONCLUSION}

In the sustainable grid the asymmetrical higher harmonics can appear even more often than symmetrical ones because the number of one phase loads is higher than three phase ones. Hence, the compensation of asymmetrical harmonics should be taken into account in research process of the grid connected AC-DC GCC. Based on the obtained results it is obvious that compensation of asymmetrical harmonics is less efficient than symmetrical one. Despite of enabled compensation process the current is more distorted $\left(\mathrm{THD}_{\mathrm{I}}=2.5 \%\right)$ with asymmetrical harmonics in comparison to $\mathrm{THD}_{\mathrm{I}}=0.7 \%$ for symmetrical harmonics with the same level $\mathrm{THD}_{\mathrm{U}}=20 \%$. The research process is in progress. Therefore, in this paper some kind of introduction to the problem is presented. The final solution to obtain satisfactory results with asymmetrical higher harmonics distortions has to be developed. However, it should be stressed that for considered conditions, developed compensation algorithm works very well - current $\mathrm{THD}_{\mathrm{I}}$ is reduced from $50 \%$ to $3 \%$ in laboratory conditions (Fig.14 and Fig. 15).

\section{ACKNOWLEDGMENT}

This work has been partially supported by the National Center for Research and Development, Poland, developing grant no. NR01 0014 06/2009.

This work has been partially supported by the European Union in the framework of European Social Fund through the Warsaw University of Technology Development Program, realized by Center for Advanced Studies.

\section{REFERENCES}

[1] Meyer, C.; Hoing, M.; Peterson, A.; De Doncker, R.W. Control and design of DC grids for offshore wind farms. IEEE Trans. Industry Appl. 2007, 43, 1475-1482.

[2] Ł. H. Kocewiak, "Harmonics In Large Offshore Wind Farms", PhD Thesis 2012, Aalborg, Denmark.

[3] M. Malinowski, M. Jasinski, and M. P. Kazmierkowski, "Simple Direct Power Control of three-Phase PWM Rectifier Using Space-Vector Modulation (DPC-SVM)," IEEE Transaction on Industrial Electronics, vol. 51, No. 2, April 2004, pp. 447- 454.

[4] V. Ignatova, P. Granjon, S. Bacha i F. Dumas "Classification and characterization of three phase voltage dips by space vector methodology", FPS, Amsterdam, Netherlands, 2005, pp. 1-6.

[5] European Standard EN-50160, "Voltage characteristics of electricity supplied by public distribution systems", CENELEC, Brussels, Belgium, 1994.

[6] M. P. Kazmierkowski, R. Krishnan, F. Blaabjerg, Control in Power Electronics, New York, Academic, 2002

[7] Kazmierkowski M.P., Jasinski M., Wrona G., "DSP-Based Control of Grid-Connected Power Converters Operating Under Grid Distortions", IEEE Transactions on Industrial Informatics, $\mathrm{Nr} 7 / 2011$, pages 204 211, ISSN : 1551-3203.

[8] Paolo Mattavelli, Fernando Pinhabel Marafão; "Repetitive-Based Control for Selective Harmonic Compensation in Active Power Filter"; IEEE Transaction on Industrial Electronics, vol. 51, No. 5, October 2004.

[9] R. Teodorescu, F. Blaabjerg, M. Liserre, and P.C. Loh, "Proportional resonant controllers and filters for grid-connected voltage-source converters," Electric Power Applications, IEE Proceedings, vol. 153, no.5, pp. 750-762, Sept. 2006.

[10] Yuan, X., Merk, W., Stemmler, H., and Allmeling, J., "Stationary frame generalized integrators for current control of active power filters with zero steady-state error for current harmonics of concern under unbalanced and distorted operating conditions," IEEE Trans. Ind. Appl., vol. 38, no.2, pp. 523-532, Mar./Apr. 2002.

[11] H. Akagi, E. H. Watanabe, M. Aredes, Instantaneous Power Theory and Applications to Power Conditioning, IEEE-Press and J. Wiley\& Sons, Inc., 2007.

[12] H. Akagi, "Modern active filters and traditional passive filters," Bulletin of the Polish Academy of Sciences, Technical Sciences, Vol. 54, No. 3, 2006, pp. 255-269.

[13] Limongi, L.R.; Bojoi, R.; Pica, C.; Profumo, F.; Tenconi, A. "Analysis and Comparison of Phase Locked Loop Techniques for Grid Utility Applications", Power Conversion Conference - Nagoya, 2007. PCC '07, On page(s): $674-681$

[14] D. N. Zmood and D. G. Holmes, "Stationary frame current regulation of PWM inverters with zero steady state error," in Proc. IEEE PESC, 1999,pp. 1185-1190.

[15] M. Liserre, R. Teodorescu, and F. Blaabjerg, "Multiple Harmonics Control for Three-Phase Grid Converter Systems With the Use of PIRES Current Controller in a Rotating Frame", IEEE Trans. Power Electr., Vol. 21, May 2006.

[16] C. L. Fortescue, "Method of symmetrical coordinates applied to the solution of polyphase networks," Trans. of the AIEE, vol. 37, Part II, pp. 1027-1140, July 1918.

[17] W. V. Lyon, Application of the Method of Symmetrical Components. New York: McGraw-Hill, 1937. 


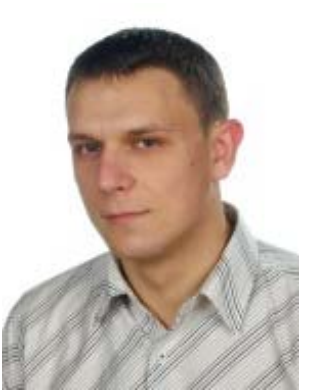

Grzegorz Wrona received the M.Sc.E.E. degree in electrical engineering from the Institute of Control and Industrial Electronics, Warsaw University of Technology (WUT), Warsaw, Poland, in 2010.

Since 2010 he is a Ph.D. student in Electrical Engineering Faculty, WUT. His research interests include power converters applied to renewable energy applications, control of power electronic converters and industrial drives, power quality in renewable generation plants and DSP Microcontroller.

E-mail: grzegorz.wrona@ee.pw.edu.pl

Postal address: Institute of Control and Industrial Electronics, Warsaw University of Technology, ul. Koszykowa 75, 00-662 Warszawa, Poland.

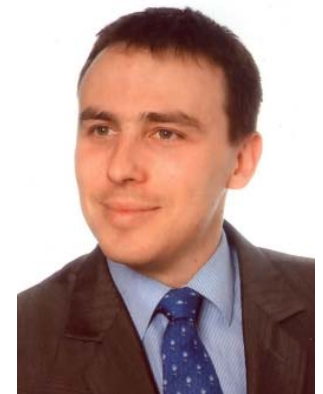

Marek Jasinski (M'00 SM'12) received the M.Sc.E.E. degree with SEP and IEEE (PS) distinction, and Ph.D. degree in Electrical Engineering from the Institute of Control and Industrial Electronics (ICIE), Warsaw University of Technology (WUT), Warsaw, Poland, in 2000, and in 2005, respectively. Since 2006 he is with the ICIE, WUT as Assistant Professor. His research activity deals with control of power electronics converters for drives and renewable energy sources. Since 2006 he is working on power take off train for renewable energy sources. He is an author or co-author more than 60 technical papers. He was at Aalborg University, Denmark, as a guest researcher of the VESTAS Power Program, in 2009. Dr Jasinski is a IEEE PS Chapters Coordinator. He was a scholar of Foundation for Polish Science and Center for Advanced Studies of WUT. He received ABB distinction for his Ph.D. dissertation, and SIEMENS Research Award.

E-mail: mja@isep.pw.edu.pl

Postal address: Institute of Control and Industrial Electronics, Warsaw University of Technology, ul. Koszykowa 75, 00-662 Warszawa, Poland. 